\title{
Effects of Head Direction on Electromyographic Activity of Quadriceps, Center of Pressure and Foot Pressure during Squat Exercise
}

\author{
Yao Xue, PT • Kyu-Ryeong Kim, PT, MS • Myoung-Kwon Kim, PT, $\mathrm{PhD}^{1 \dagger}$ \\ Department of Rehabilitation Sciences, Graduate School, Daegu University, \\ ${ }^{1}$ Department of Physical Therapy, College of Rehabilitation Sciences, Daegu University
}

Received: October 29, 2020 / Revised: November 23, 2020 / Accepted: January 19, 2021

(C) 2021 J Korean Soc Phys Med

\begin{abstract}
| Abstract |
PURPOSE: This study examined the effects of changes in the head direction (forward, upward $10^{\circ}$ and downward $10^{\circ}$ ) on the quadriceps, center of pressure (COP), and foot pressure during squat exercises. The aim was to determine if the head direction could better activate the quadriceps muscle and provide a safer and stable squat posture during squat exercise.
\end{abstract} METHODS: Fifteen healthy college students were asked to stand on a Zebris, and three electrodes for sEMG were attached to their vastus medialis oblique (VMO), vastus lateralis (VL), and rectus femoris (RF) muscles. The participants then performed squatting exercises under three head directions (forward, upward $10^{\circ}$, and downward $10^{\circ}$ ). Surface electrodes were then used to record the EMG data during exercise. The Zebris FDM-SX was used to measure the foot pressure and COP of the participants.

RESULTS: In squat exercise, the upward head direction group showed significantly higher VL activation than the downward head direction group $(\mathrm{p}<.05)$. The upward head

$\uparrow$ Corresponding Author : Myoung-Kwon Kim skybird-98@hanmail.net, https://orcid.org/0000-0002-7251-6108 This is an Open Access article distributed under the terms of the Creative Commons Attribution Non-Commercial License (http://creativecommons.org/licenses/by-nc/3.0) which permits unrestricted non-commercial use, distribution, and reproduction in any medium, provided the original work is properly cited. direction group showed a significant backward change in the deviation of the COP than the downward and forward groups $(\mathrm{p}<.05)$. The upward head direction group showed a significant decrease in forefoot pressure than the downward and forward groups $(\mathrm{p}<.05)$ and an increase in the hindfoot pressure compared to the downward and forward groups $(p<.05)$.

CONCLUSION: The head direction upward in squat exercise has a positive effect on the quadriceps.

Key Words: Electromyography, Exercise, Quadriceps muscle

\section{Introduction}

In recent years, as office activities have increased and outdoor time has decreased, people's health has become problematic. The squat is an exercise that indirectly improves the quality of life of sports and non-sports people by increasing the strength of the hip and knee extensors [1]. The squat is related to many daily activities, such as lifting heavy objects, because it can recruit multiple muscle groups in a single movement [3]. It is also included in many sports to improve athletic performance as a core sport [4]. The squat exercise mainly strengthens the lower body muscles and core muscles, including the quadriceps, biceps femoris, gluteus maximus, and gastrocnemius [6]. 
Considerable research has been conducted to improve the posture of squat exercise in strengthening the quadriceps muscles $[5,6]$.

The correctness of the squat is essential for preventing injuries. The spine is a whole unit, and changes in one place may cause compensation activities in other parts. Therefore, incorrect head movements can cause incorrect body compensation, resulting in injury $[2,7,8]$. Changes in the gaze direction and head position can affect the spine kinematics [1]; there have been many discussions on the expected effect and safety of squat exercise. Most existing research on squat exercise have either studied the degree of activation of the lower extremity muscles through different ways of foundation planes $[9,10]$ or have studied the relationship between the degree of activation of the lower extremity muscles and the position changes of knee or ankle joints [11-13]. Some papers reported the effects of the torso and upper limb position on the lower limb muscles during squat exercise [14-17].

In squat exercise, however, the direction of gaze or head position is still controversial. Myer et al. reported that maintaining the neutral alignment of the head, neck, and torso during squatting is an important aspect [18]. Looking upwards during squat exercise appears to have a more positive effect [19]. Donnelly et al. discussed the effects of an upward, forward, and downward gaze on the body kinematics during squat exercise. They reported that downward gaze would increase the degree of hip flexion. Therefore, the head or gaze direction of athletes should not be lower than the neutral direction [8]. In squat exercise, an increased and faster center of pressure (COP) displacement may also increase the risk of injury to the athlete [20]. Yoon et al. reported that in a squat, the COP could strengthen the quadriceps and gluteus maximus on the hindfoot [21]. The forward shift COP resulted in higher loads for the "posterior muscles" [22].

No study has compared the activation of quadriceps muscle according to the head direction after maintaining the knee joint during squat exercise. Based on the above research, it was hypothesized that during squat exercises, the muscle activity of the quadriceps, COP, and foot pressure would be affected when the head position changes.

\section{Methods}

\section{Participants}

The participants in this study were 15 healthy Daegu University students aged between 20 and 30 years. The ethical committee of Daegu University approved this study, and the IRB number was 1040621-202007-HR-015.

The inclusion criteria of the participants were 1) no visual or vestibular system injuries, 2) no back pain, lower limb injuries, and 3) no musculoskeletal injuries. The exclusion criteria of participants were 1) have done other sports or exercises before and after the experiment, 2) consumed alcohol during the experiment, and 3) had diseases related to the muscle to be measured (VMO, VL, and RF).

\section{Experimental procedures}

In this study, the muscles on the right side of the participant were tested uniformly. During squat exercise, surface electromyography (EMG) data were collected using a Desktop DTS (Noraxon USA Inc, Scottsdale, AZ, USA). The EMG was attached to the vastus medialis oblique, vastus lateralis, and rectus femoris. The Zebris FDM-SX (zebris Medical GmbH, Germany) was used to measure the foot pressure and COP of the participants. During the squat exercise, the participants stood in front of a mirror wall (approximately 1.6 meters away from the mirror) and performed three sets of exercises under three different conditions. Condition A requires the participant to stare straight ahead and maintain a neutral head position. Condition B requires the participant to extend their head extension $10^{\circ}$, keeping the direction of the head and line of sight consistent. Condition $\mathrm{C}$ required the participant 


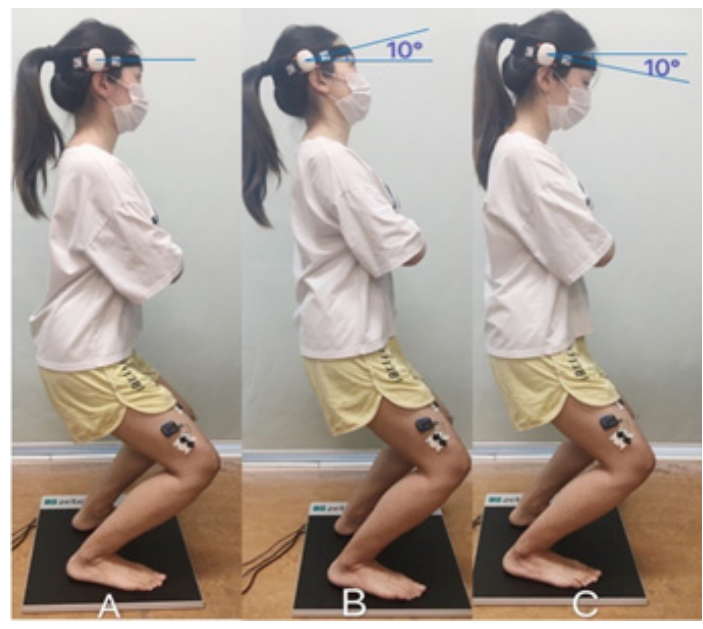

Fig. 1. Three sets of squat exercises: A. Forward head direction B. Upward head direction C. Downward head direction.

to bend their head flexion $10^{\circ}$, keeping the direction of the head and line of sight consistent (Fig. 1). The change in head angle was controlled using BPMpro Technology (Brown Spring, USA).

Before the experiment, each participant placed three cards randomly. They then turned the cards over and performed three sets of squat training in the order of the cards. For the squat posture, to facilitate the foot pressure and COP measurements, the participant stood on a fixed position on Zebris, and the trunk was maintained in a neutral position. The knee flexion angle was set to $90^{\circ}$ to avoid fatigue caused by frequent squatting of participants and affect the experimental results. Each set of actions lasted for five seconds. The participant then stood up slowly and repeated this action three times. A 30 -second rest was allowed between each measurement. The participants were given two minutes of rest after each change in head direction. BPMpro Technology was used to measure the knee flexion angle in the experiment. Each participant was asked to maintain a neutral torso so that the hip joint angle would be the same.

\section{Intervention}

During squat exercise, the torso must be maintained in a neutral position. The experimenter needed to ensure that the participant's knee flexion angle was the same every time they performed the squat. The participant's head movement should be consistent with the head direction. In the event of an error, the measurement needed to be retaken. The EMG, COP, and foot pressure were measured simultaneously.

\section{Measurement}

1) Surface Electromyography (EMG)

A Desktop DTS (Noraxon USA INC, Scottsdale, AZ, USA) was used to collect and analyze the surface EMG data. The sampling rate was $1000 \mathrm{~Hz}$, and the band-pass filter frequency was $20 \mathrm{~Hz}$ to $500 \mathrm{~Hz}$. The EMG data processing in this study is the root mean square (RMS) value calculated based on a 50-millisecond data point window. The EMG signal of each action was collected for five seconds. The data of the first and last second was deleted, and only three seconds were analyzed. The EMG was measured three times, and the average was taken [23]. The participants could rest for two minutes between each measurement. All EMG data were the average of three replicates. The experimental data are expressed as a percentage of the calculated RMS of the MVIC (\%MVIC).

The electrodes were placed parallel to the muscle fibers, with the centers $2.5 \mathrm{~cm}$ [24]. Before connecting the electrodes, the skin was cleaned with alcohol swabs. The VMO electrodes were aligned $60^{\circ}$ medially from the longitudinal axis of the femur. The VL electrodes were aligned $15^{\circ}$ laterally from the longitudinal axis of the femur [25]. The RF electrodes were placed on the anterior surface of the thigh. To standardize the EMG signal of the muscle, the RMS of the maximum voluntary isometric contraction (MVIC) for five seconds was calculated for each muscle at the appropriate manual muscle test position [26]. 
Table 1. General Participant Characteristics $(n=15)$

\begin{tabular}{cc}
\hline Variable & Participants \\
\hline Age (year) & $25.69 \pm 3.59^{\alpha}$ \\
Height $(\mathrm{cm})$ & $171.15 \pm 8.68$ \\
Weight $(\mathrm{kg})$ & $64.87 \pm 10.80$ \\
BMI & $21.97 \pm 1.85$ \\
\hline
\end{tabular}

${ }^{\alpha}$ Mean \pm SD, BMI: Body Mass Index

2) Zebris FDM-SX (zebris Medical GmbH, Germany)

The COP and foot pressure parameters were obtained from Zebris FDM-SX (zebris Medical GmbH, Germany) during the squat exercise. The device consisted of multiple miniature force sensors. When the participant was standing on Zebris, the sensors recorded the force exerted by the foot at a sampling rate of $120 \mathrm{~Hz}$. The data of the three sets of movements were obtained simultaneously by EMG, and the start and end times of each movement were the same. The front and back deviation of the COP could be derived from the Y-axis deviation on Zebris.

\section{Statistical Analysis}

All data collected in this study were performed using SPSS (IBM Corp. Released 2012. IBM SPSS Statistics for Windows, Version 21.0. Armonk, NY: IBM Corp). A one-way repeated measures ANOVA was performed to compare the activation levels of the three muscles examined across the three squat exercises. The LSD test was used to correct for multiple comparisons. A p-value $<.05$ indicated a significant difference in the changes of the quadriceps, $\mathrm{COP}$, and foot pressure in the three exercise groups.

\section{Results}

\section{General Participant Characteristics}

Table 1 summarizes the clinical and demographic features of the study participants $(n=15)$.

\section{Changes in EMG activation of Quadriceps}

A significant difference in the vastus lateralis activation according to three different head directions was observed $(\mathrm{p}<.05)$ (Table 2). Compared to the downward head direction, activation of the vastus lateralis increased significantly with the upward head gaze $(\mathrm{p}<.05)$ (Table 2$)$. The vastus medialis oblique and rectus femoris activations were similar in the three different head directions $(p>$ .05) (Table 2).

3. Changes in the deviation of the COP on the $Y$-axis

A significant difference in the deviation of $\mathrm{COP}$ on the Y-axis was observed according to three different head directions $(\mathrm{p}<.05)$ (Table 3). Compared to the forward head direction, the COP moved significantly backward with the upward head, and the COP moved significantly forward with the downward head $(\mathrm{p}<.05)$ (Table 3).

\section{Changes in Foot Pressure}

A significant difference in foot pressure according to three different head directions was noted $(\mathrm{p}<.05)$ (Table 4). Compared to the forward head direction, the foot pressure on the hindfoot increased significantly with the upward head direction $(\mathrm{p}<.05)$ (Table 4). Compared to

Table 2. EMG Activation of the Quadriceps According to the Three Head Directions $(n=15)$ (Unit: \%MVIC)

\begin{tabular}{ccccccc}
\hline Variable & FG & UG & DG & F & p & Post-hoc \\
\hline Vastus medialis oblique & $66.41 \pm 23.9^{\alpha}$ & $77.44 \pm 30.80$ & $58.38 \pm 21.54$ & 2.091 & .136 & \\
Vastus lateralis & $65.58 \pm 17.56$ & $77.62 \pm 20.71$ & $58.18 \pm 17.58$ & 4.208 & $.022^{*}$ & UG $>$ DG \\
Rectus femoris & $59.31 \pm 21.28$ & $73.11 \pm 25.45$ & $56.62 \pm 21.66$ & 2.303 & .112 & \\
\hline
\end{tabular}

${ }^{\alpha}$ Mean \pm SD, FG: Forward Group, UG: Upward Group, DG: Downward Group, ${ }^{*} p<.05$ 
Table 3. Deviation of the COP on the $Y$ Axis According to Three Head Directions $(n=15)$ (Unit: $\mathrm{mm})$

\begin{tabular}{ccccccc}
\hline Variable & FG & UG & DG & F & p & Post-hoc \\
\hline & & & & & DG $>$ FG, \\
Deviation of COP & $11.92 \pm 25.50^{\alpha}$ & $-12.02 \pm 32.86$ & $32.54 \pm 21.97$ & 10.291 & $.000^{*}$ & DG $>$ UG, \\
& & & & & FG $>$ UG \\
\hline
\end{tabular}

${ }^{\alpha}$ Mean \pm SD, FG: Forward Group, UG: Upward Group, DG: Downward Group, ${ }^{*} p<.05$

Table 4. Foot Pressure According to the Three Different Head Directions ( $n=15)$ (Unit: $N$ )

\begin{tabular}{|c|c|c|c|c|c|c|}
\hline Variable & FG & UG & DG & $\mathrm{F}$ & $\mathrm{p}$ & Post-hoc \\
\hline Right forefoot pressure & $54.98 \pm 21.81^{\alpha}$ & $40.14 \pm 16.44$ & $71.18 \pm 11.29$ & 10.283 & $.000^{*}$ & $\begin{aligned} \mathrm{FG} & >\mathrm{UG} \\
\mathrm{DG} & >\mathrm{FG} \\
\mathrm{DG} & >\mathrm{UG}\end{aligned}$ \\
\hline Right hindfoot pressure & $45.05 \pm 21.78^{\alpha}$ & $59.86 \pm 16.44$ & $29.01 \pm 10.72$ & 10.284 & $.000^{*}$ & $\begin{aligned} \mathrm{UG} & >\mathrm{FG} \\
\mathrm{UG} & >\mathrm{DG} \\
\mathrm{FG} & >\mathrm{DG}\end{aligned}$ \\
\hline Left forefoot pressure & $57.03 \pm 16.38^{\alpha}$ & $35.08 \pm 16.18$ & $70.03 \pm 14.80$ & 18.193 & $.000 *$ & $\begin{aligned} \mathrm{DG} & >\mathrm{FG} \\
\mathrm{DG} & >\mathrm{FG} \\
\mathrm{FG} & >\mathrm{UG}\end{aligned}$ \\
\hline Left hindfoot pressure & $42.97 \pm 16.38^{\alpha}$ & $65.40 \pm 16.31$ & $29.70 \pm 14.47$ & 18.685 & $.000 *$ & $\begin{aligned} \mathrm{UG} & >\mathrm{FG} \\
\mathrm{UG} & >\mathrm{DG} \\
\mathrm{FG} & >\mathrm{DG}\end{aligned}$ \\
\hline
\end{tabular}

${ }^{\alpha}$ Mean \pm SD, FG: Forward Group, UG: Upward Group, DG: Downward Group, ${ }^{*} p<.05$

the forward head direction, the foot pressure of the forefoot was significantly higher than with the downward head direction $(\mathrm{p}<.05)($ Table 4$)$

\section{Discussion}

Squat training is a physical activity that individuals can easily perform at a relatively low cost. The exercise is performed widely by the public to keep fit and promote health, and professional athletes also use it extensively to improve their performance $[27,28]$. To strengthen the quadriceps exercise, people use many squat positions for exercise. By changing the squat angle, the standing plane, or the distance between the feet, few people have explored the impact of changes in the head position on the squat exercise.
This study found that the normalized EMG activity in the VL significantly differed according to the three head directions. Compared to the forward head direction, the VL activity increased by approximately $18.4 \%$ when the head direction was upward. When the head direction was downward, it decreased by approximately $11.3 \%$. Although there was no significant difference in the activity of the VMO and RF, the muscle activity increased while gazing upward. Compared to the forward head direction, the VMO activity increased by approximately $16.6 \%$ when the head direction is upward. When the head direction was downward, it decreased by approximately $12.1 \%$. Compared to the forward head direction, the RF activity increased by about $23.3 \%$ when the head direction was upward. When the head direction is downward, it decreased by approximately $4.5 \%$. This study also found a significant 
difference in foot pressure according to three different head directions. Compared to the downward head direction, the COP moves toward the hindfoot, and the foot pressure of the hindfoot increased when the head direction is upward. When the head direction was downward, the COP moved to the front foot, and the foot pressure of the forefoot increased.

This finding is of great significance for muscle activation of the quadriceps during squatting exercises. The results show that the head direction needs to be placed upward to improve muscle activation of the quadriceps during the squatting process.

There are several possible explanations for these results. Dionisio et al. reported that the quadriceps muscle was strongly activated when the COP was on the forefoot with the head kept neutral. They attributed this result to the additional flexion of the ankle and knee joints causing the thighs and calves to move forward. Moreover, the plantarflexion torque of the ankle joint increased to prevent the body from falling [29]. On the other hand, this study found that the quadriceps muscle activity increased with the head direction upwards, especially the VL muscle, and the COP moved to the hindfoot. The quadriceps muscle activity decreased with the downwards head direction, and the COP moved significantly to the forefoot. The current study finding is consistent with Kitamura et al., who reported that the forward movement of the COP during squatting exercise would increase the load on the muscles of the back of the thigh significantly, resulting in a decrease in quadriceps activity [22]. Despite keeping the trunk as neutral as possible, it may still increase the potential flexion of the hips and trunk [18], resulting in a decrease in the activity of the front thigh muscles. The head extension challenged the subjects' balance ability [30]. The subjects' body swing increased, and the back muscle activity increased [31]. At the same time, to maintain the posture and avoid falling, the body was resisting the force of falling backward, and the load on the front thigh muscles increased.
The COP moving backward during squatting increases the rectus femoris activity [21]. In conclusion, when the head was moved forward, a bowstringing force in the knee joint increased due to difficulty maintaining balance, and the VL muscle activation was higher than that of RF and VMO.

This paper only reports the results of healthy college students. For people who want to activate the quadriceps through squatting exercise, it is an excellent choice to keep the head upward while squatting. Keep the COP on the hind feet. It also seems to be more conducive to the activation of the quadriceps. The angle of the squat is also very important. This study was conducted with a knee joint angle of $90^{\circ}$. Different knee flexion angles can affect the activity of the quadriceps. Squat training is not only used in exercise programs for athletes or healthy people; it is also included in programs to treat musculoskeletal diseases, such as patellofemoral pain syndrome and other hip, knee, and ankle dysfunctions [4]. Among these squatting postures designed to treat musculoskeletal diseases, the recovery of the medialis oblique muscle function is considered the most important rehabilitation goal [32]. The results of this study showed that the activation of the vastus lateralis was different. Although the activity of vastus medialis oblique and rectus femoris increased, there was no significant difference. The head extension will also increase the instability of the body. Although the unstable body state is more conducive to muscle exercise [33], it increases the risk of falling. Therefore, in squat training, an upward head gaze may not be a good choice for people with musculoskeletal diseases. The conclusions of this study may help rehabilitation practitioners adjust the use of squatting exercises to improve the motor function.

\section{Conclusion}

The muscle activity of quadriceps, COP, and foot pressure according to three different head directions of 15 healthy university students were compared to determine 
the most effective head direction for muscle activation of the quadriceps. A significant difference in the vastus lateralis under upward head direction compared to downward head direction was noted. Furthermore, there were significant differences in changes in the $\mathrm{COP}$ and foot pressure in the upward head direction. Therefore, this study suggests that in squat exercise, the upward head direction has a positive effect on the muscle activity of the VL muscle.

\section{References}

[1] Schoenfeld BJ. Squatting kinematics and kinetics and their application to exercise performance. J Strength Cond Res. 2010;24(12):3497-506.

[2] Fry AC, Smith JC, Schilling BK. Effect of knee position on hip and knee torques during the barbell squat. J Strength Cond Res. 2003;17(4):629-33.

[3] Escamilla RF, Fleisig GS, Zheng N, et al. Effects of technique variations on knee biomechanics during the squat and leg press. Med Sci Sports Exerc. 2001; 33(9):1552-66.

[4] Escamilla RF. Knee biomechanics of the dynamic squat exercise. Med Sci Sports Exerc. 2001;33(1):127-41.

[5] Signorile JF, Kwiatkowski K, Caruso JF, et al. Effect of foot position on the electromyographical activity of the superficial quadriceps muscles during the parallel squat and knee extension. J Strength Cond Res. 1995;9(3):182-7.

[6] Boyden G, Scurr J, Dyson R. A comparison of quadriceps electromyographic activity with the position of the foot during the parallel squat. J Strength Cond Res. 2000;14(4):379-82.

[7] Allum J, Gresty M, Keshner E, et al. The control of head movements during human balance corrections. J Vestib Res. 1997;7(2-3):189-218.

[8] Donnelly DV, Berg WP, Fiske DM. The effect of the direction of gaze on the kinematics of the squat exercise.
J Strength Cond Res. 2006;20(1):145-50.

[9] Hyong IH, Kang JH. Activities of the vastus lateralis and vastus medialis oblique muscles during squats on different surfaces. J Phys Ther Sci. 2013;25(8):915-7.

[10] Cho M, Kang JY, Oh JH, et al. The effects of performing squats on an inclined board on thigh muscle activation. Phys Ther Rehabil Sci. 2017;6(1):39-44.

[11] de Oliveira Sousa C, de Almeida Ferreira JJ, Medeiros ACLV, et al. Electromyograhic activity in squatting at 40, 60 and 90 knee flexion positions. Rev Bras Med Esporte. 2007;13(5):280-6.

[12] Macrum E, Bell DR, Boling M, et al. Effect of limiting ankle-dorsiflexion range of motion on lower extremity kinematics and muscle-activation patterns during a squat. J Sport Rehabil. 2012;21(2):144-50.

[13] Schütz P, List R, Zemp R, et al. Joint angles of the ankle, knee, and hip and loading conditions during split squats. J Appl Biomech. 2014;30(3):373-80.

[14] Kulas AS, Hortobágyi T, DeVita P. Trunk position modulates anterior cruciate ligament forces and strains during a single-leg squat. Clin Biomech. 2012;27(1): 16-21.

[15] Lee TS, Song MY, Kwon YJ. Activation of back and lower limb muscles during squat exercises with different trunk flexion. J Phys Ther Sci. 2016;28(12):3407-10.

[16] Glave AP, Olson JM, Applegate DK, et al. The effects of two different arm positions and weight status on select kinematic variables during the bodyweight squat. $\mathrm{J}$ Strength Cond Res. 2012;26(11):3148-54.

[17] Takahashi K, Yamaji T, Wada N, et al. Trunk kinematics and muscle activities during arm elevation. J Orthop Sci. 2015;20(4):624-32.

[18] Myer GD, Kushner AM, Brent JL, et al. The back squat: a proposed assessment of functional deficits and technical factors that limit performance. Strength Cond J. 2014;36(6):4-27.

[19] O'Shea P. Sports performance series: The parallel squat. Strength Cond J. 1985;7(1):4-6. 
[20] Sibley KM, Straus SE, Inness EL, et al. Clinical balance assessment: perceptions of commonly-used standardized measures and current practices among physiotherapists in Ontario, Canada. Implementation Sci. 2013;8(1):33.

[21] Yoon WR, Park SH, Jeong CH, et al. Effects of center of pressure on muscle activations and joint range of motion of lower extremities during squat. Korean journal of Sport Biomechanics. 2018;28(1):37-43.

[22] Kitamura T, Kido A, Ishida Y, et al. Muscle activity pattern with a shifted center of pressure during the squat exercise. J Sports Sci Med. 2019;18(2):248-52.

[23] Reinold MM, Wilk KE, Fleisig GS, et al. Electromyographic analysis of the rotator cuff and deltoid musculature during common shoulder external rotation exercises. J Orthop Sports Phys Ther. 2004;34(7):385-94.

[24] De Luca CJ. The use of surface electromyography in biomechanics. J Appl Biomech. 1997;13(2):135-63.

[25] Rice MA, Bennett JG, Ruhling RO. Comparison of two exercises on VMO and VL EMG activity and force production. Isokinet Exerc Sci. 1995;5(2):61-7.

[26] Kendall FP, McCreary EK, Provance PG, et al. Muscles, testing and function: with posture and pain (5th ed). Baltimore. Lippincott, Williams \& Wilkins. 2005.

[27] Chae WS, Jeong HK, Jang JI. Effect of different heel plates on muscle activities during the squat. Korean Journal of Sport Biomechanics. 2007;17(2):113-21.

[28] Back SG. Effects of using convergence circuit weight training on the blood lipids and oxygen-carrying factors in middle-aged women. Journal of the Korea Convergence Society. 2016;7(6):267-74.

[29] Dionisio VC, Almeida GL, Duarte M, et al. Kinematic, kinetic and EMG patterns during downward squatting. J Electromyogr Kinesiol. 2008;18(1):134-43.

[30] Kogler A, Lindfors J, Ödkvist L, et al. Postural stability using different neck positions in normal subjects and patients with neck trauma. Acta Otolaryngol. 2000; 120(2):151-5.

[31] Barin K, Seitz CM, Welling DB. Effect of head orientation on the diagnostic sensitivity of posturography in patients with compensated unilateral lesions. Otolaryngol Head Neck Surg. 1992;106(4):355-62.

[32] Crossley K, Bennell K, Green S, et al. A systematic review of physical interventions for patellofemoral pain syndrome. Clin J Sport Med. 2001;11(2):103-10.

[33] Andersen V, Fimland M, Brennset O, et al. Muscle activation and strength in squat and Bulgarian squat on stable and unstable surface. Int $\mathrm{J}$ Sports Med. 2014;35(14):1196-202. 